\title{
Sicher und schnell für Behandler und Patienten
}

Ein toller Service für die Patienten, und in dringenden Fällen mehr als das: einfach in die Praxis zu kommen und sie mit einer fertigen Restauration wieder zu verlassen. Dafür gibt es CEREC. Es ist die einzige ausgereifte Methode in der Zahnheilkunde, die es ermöglicht, eine Krone, Teilkrone, ein Inlay oder Veneers in nur 1 Sitzung herzustellen.

CEREC steht für die Digitalisierung der Zahnarztpraxis: Mit dem 1. CAD/CAMSystem kam 1985 das 3-dimensionale Scannen des Patientengebisses in die Zahnheilkunde und führte zu vielen technologischen Innovationen. Die digitale Abformung sorgte nicht nur in der Prothetik für einen enormen Entwicklungsschub, sondern auch in der Kieferorthopädie sowie in der Implantologie.

Doch CEREC ist weit mehr als ein „GebissScan“ - es ist ein integriertes, digitales System. Es beginnt bei der digitalen Abformung mit der CEREC Omnicam, der kleinsten puderfrei arbeitenden Farb-Videokamera auf dem Markt. Es folgt die Konstruktion der Restauration mit der neuen CEREC SW 4.4, in die mit Biokiefer eine Anwendung integriert ist, die hervorragende Erstvorschläge auf der Basis des gesamten gescannten Bereichs entwickelt. Mit der Artikulator-Funktion lassen sich neben den statischen auch die dynamischen Kontaktflächen ermitteln und

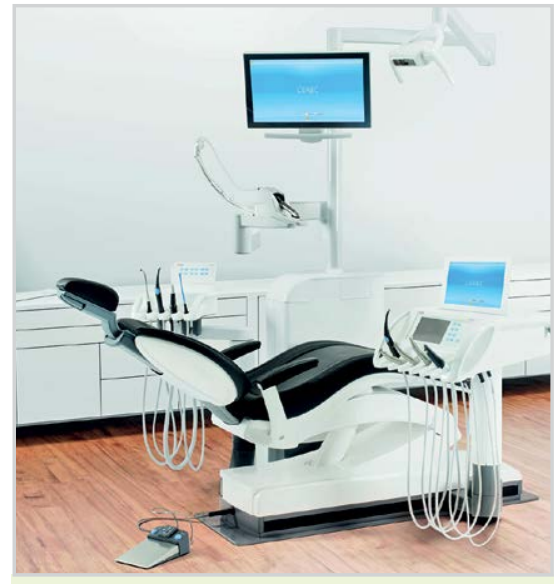

CEREC AI: Die CEREC Omnicam lässt sich fast wie ein Winkelstück direkt an der Behandlungseinheit nutzen. somit die korrekte funktionelle Okklusion bestimmen. Unmittelbare Vorteile: große Natürlichkeit selbst unterhalb des Zahnäquators, kaum notwendige Nacharbeiten. Die Herstellung der geplanten Restauration mit 3 möglichen CEREC-Fertigungsmaschinen komplettiert das System.

\section{Komplett versorgt in nur 1 Sitzung}

Auf diese Weise profitieren sowohl Behandler als auch Patienten: Zum einen behält der Zahnarzt über den gesamten Prozess die Kontrolle. Zum anderen erlebt der Patient eine Behandlung ohne unangenehme Löffel-Abformung. Er wird nur einmal anästhesiert, er benötigt kein Provisorium und verlässt die Praxis nach maximal 90 min final versorgt. Das ist in der Zahnheilkunde bis heute mit keinem anderen System möglich. Die Erfahrungen zeigen: Patienten sind von der Technik fasziniert. Sie fassen Vertrauen zu ihrem Zahnarzt und sind eher bereit, vorgeschlagene Therapien zu akzeptieren. Inzwischen wird alle $5 \mathrm{~s}$ irgendwo auf der Welt eine CEREC-Restauration eingesetzt - in Deutschland steht mittlerweile in jeder 7 . Praxis solch ein CAD/CAM-System für die Chairside-Versorgung in einer Sitzung.

Doch längst dient dieses CAD/CAM-System nicht mehr nur dazu, Restaurationen herzustellen. In CEREC lassen sich die 3DRöntgendaten mit dem prothetischen Vorschlag zusammenführen, um beispielsweise für die Implantat-Insertion eine Bohrschablone zu planen und sogar in weniger als $1 \mathrm{~h}$ selbst herzustellen (CEREC Guide 2) - das gelingt so mit keinem anderen System. Einzelzahn-Implantationen können auf diese Weise sicher durchgeführt und gegebenenfalls auch in nur 1 Sitzung final versorgt werden.

Außerdem ermöglicht die neue CEREC Ortho Software einen sicheren geführten Scan mit der CEREC Omnicam, um ein digitales Modell des gesamten Kieferbogens erstellen. Diese Daten können dann für die Planung kieferorthopädischer Behand-

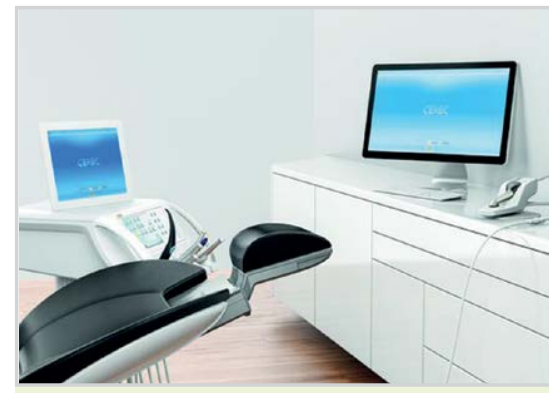

CEREC AF: Die CEREC Omnicam für die flexible Nutzung in der gesamten Praxis, selbst wenn sie sich über mehrere Etagen erstreckt.

lungen und die Herstellung dafür erforderlicher Apparaturen mit nur einem Klick versendet werden.

Vom Zusammenführen von Daten aus verschiedenen diagnostischen Verfahren in 1 System profitiert eine Zahnarztpraxis gleich mehrfach. Die Abläufe werden einfacher, lassen sich besser nachvollziehen und kontrollieren. Die Sicherheit und Qualität steigt durch die Wiederholbarkeit von Prozessen, etwa der digitalen Abformung. Das bedeutet letztlich auch eine größere Wirtschaftlichkeit. Und schließlich: Die Patienten sind höchst zufrieden. Sie kommen wieder und empfehlen weiter. Nach nur 1 Sitzung!

Ein Beitrag der

Sirona Dental GmbH, Bensheim

CEREC Al: Von vielen CEREC-Anwendern gewünscht ist sie jetzt Realität: Die CEREC Omnicam, die sich nahezu wie ein Winkelstück direkt an der Behandlungseinheit nutzen lässt. CEREC AI (Acquisition Integrated) besteht aus einer CEREC Omnicam mit Ablage, die in die Behandlungseinheit TENEO integriert ist, und aus einem PC mit einem separaten 24“-Monitor. Patient und Behandler können alles direkt am Monitor der Behandlungseinheit oder auf einem mitgelieferten Tablet verfolgen.

CEREC AF: Für eine Praxis mit mehreren Behandlungsräumen, die sich vielleicht auch über mehrere Etagen verteilen, bietet CEREC AF (Acquisition Flexible) eine komfortable Lösung. Diese Einheit besteht aus der CEREC Omnicam mit Ablage sowie einem PC mit 24“-Monitor. Die Kamera kann flexibel innerhalb der Praxis bewegt werden - die Konstruktion der Restauration kann in einem anderen Raum stattfinden. So wird es möglich, dass in einem Zimmer konstruiert und im nächsten Raum erneut gescannt wird. 